\title{
MS07-P13 | Enzyme Activation by A Flavoprotein Redox Network
}

Hammerstad, Marta (University of Oslo, Oslo, NOR); Gudim, Ingvild (University of Oslo, Oslo, NOR); Lofstad, Marie (University of Oslo, Oslo, NOR); Kjendseth Røhr, Åsmund (Norwegian University of Life Sciences, Ås, NOR); Hersleth, Hans-Petter (University of Oslo, Oslo, NOR)

Ribonucleotide reductases (RNRs) reduce ribonucleotides to deoxyribonucleotides by employing radical chemistry. In class Ib RNRs, reduced Nrdl, a flavodoxin-like protein, is essential for the activation of a dimanganese center in the radical generating RNR $\beta$ subunit. It has been proposed that Nrdl is recycled in vivo by an Nrdl reductase, but no such reductase had been identified. Ferredoxin/flavodoxin-NADP ${ }^{+}$oxidoreductases (FNRs) are probable reductants of Nrdl. We identified three FNRs in the genome of Bacillus cereusand carried out structural and functional studies in order to characterize their ability to reduce Nrdl. By comparing reduction kinetics, binding affinities, redox potentials and 3D structure,we have shown that one FNR reduces Nrdl at a much higher rate than the two remaining FNRs. Using this FNR as an Nrdl reductase, we were also able to activate the RNR $\beta$ subunit under aerobic conditions, mimicking cellular conditions. Altogether, our observations suggest that this FNR might be the superior Nrdl reductase in vivo, and hence, an essential activator of the class Ib RNR system.

In addition to Nrdl, B. cereus encodes two flavodoxins (Flds). We have performed biochemical and structural investigations of the full FNR-Fld/Nrdl redox network, and identified that one FNR-Fld pair is more efficient than the others. By studying the interactions between proteins in flavoprotein networks, which are poorly characterised in bacteria, we aim to map defining features that govern recognition and selectivity for electron transfer between flavoproteins. 